\title{
Angiotensin II for the treatment of vasodilatory shock: enough data to consider angiotensin II safe?
}

\author{
Nina Buchtele ${ }^{1}$, Michael Schwameis ${ }^{2}$ and Bernd Jilma ${ }^{1 *}$ \\ See related research by Busse et al., https://ccforum.biomedcentral.com/articles/10.1186/s13054-017-1896-6
}

A recent structured review by Busse et al. confirmed the efficacy of angiotensin II in terms of increasing blood pressure in patients with different types of shock [1]. The majority of data for this analysis came from the recently published ATHOS-3 trial which showed that angiotensin II effectively increased blood pressure in patients with vasodilatory shock receiving high-dose vasopressors [2]. In this trial, safety was defined as the secondary outcome. Considering the similar total number of adverse events in both groups, the administration of angiotensin II seemed safe. Antonucci et al. had already raised some caution about the administration of angiotensin II [3]. In addition, we want to add some further comments about the use of angiotensin II, especially as it has recently been approved by the US Food and Drug administration.

Taking a closer look at the subgroups of adverse events, patients in the treatment group were significantly more likely to develop infection or delirium. High doses of vasopressors are known to cause mesenteric ischemia [4], which may impair intestinal mucosal barrier function resulting in transmission of enteric bacteria into the bloodstream. It would be of great interest to analyse these specific adverse events in another structured review or meta-analysis.

Furthermore, the significant decrease in the cardiovascular Sequential Organ Failure Assessment (SOFA) score in the treatment group must be interpreted cautiously. The infusion of angiotensin II probably allowed a decrease in background catecholamines and therefore a decrease in the cardiovascular SOFA score. The SOFA score accurately predicts mortality of critically ill patients, but the cardiovascular domain has its limitations [5]. It is unclear whether the cardiovascular component of the SOFA score can be used if vasopressors other than catecholamines are infused. The key issue is whether additional points for equivalent doses of angiotensin have to be included in the score. Although hypotension improved, the total score worsened to a similar extent in both groups, suggesting that functions of other organ systems may have deteriorated.

It seems that finally efforts in developing a new effective substance in refractory shock have paid off, but the safety results have to be treated with caution.

\section{Authors' response}

Laurence W. Busse and Mizra Haider Ali

It has recently been re-established that angiotensin II is effective in raising blood pressure in patients with hypotension [1, 2]. Dr. Buchtele and colleagues raise the subject of the safety of angiotensin II, which was evaluated as a secondary outcome in ATHOS-3. While there were adverse event imbalances between the angiotensin II and

\footnotetext{
* Correspondence: bernd.jilma@meduniwien.ac.at

${ }^{1}$ Department of Clinical Pharmacology, Medical University of Vienna,

Waehringer Guertel 18-20, A-1090 Vienna, Austria

Full list of author information is available at the end of the article
}

placebo groups, the total incidence was similar. More importantly, the safety of angiotensin II was recently examined in a comprehensive review of over 31,000 subjects and was shown to be well tolerated, with only two deaths and fewer than 300 subjects with adverse events reported [6]. Nonetheless, we agree that further investigation should focus on the imbalances noted in ATHOS-3 regarding safety, including delirium, infection, and thromboembolism. 
The use of the cardiovascular SOFA score as a secondary endpoint in ATHOS-3 must indeed be understood cautiously. Dr. Buchtele and colleagues rightly point out that the infusion of angiotensin II allowed a decrease in the background catecholamines resulting in a decrease in the score. This was, in fact, the sole purpose of this endpoint, which was never intended to be interpreted clinically. The cardiovascular SOFA score allowed for standardisation of different vasopressor regimens specifically to observe the sparing effect that angiotensin II had on norepinephrine equivalent vasopressors. The norepinephrine equivalence of phenylephrine and vasopressin, not included in SOFA, are best approximations of equivalence based on available medical literature, but specific equivalence conversions are not important. Rather, ATHOS-3 showed that, whatever the vasopressor cocktail, angiotensin II allowed for sparing of catecholamines, which are known to be associated with mortality and adverse events $[7,8]$. The message elucidated by this end-point is that a balanced approach, using lower doses of vasopressors with different mechanisms of action, effectively raises blood pressure and may avoid some of the toxicities associated with high doses of single agent therapy.

Finally, just as the cardiovascular SOFA should be interpreted with caution, so too should the total SOFA score. While total SOFA score increased in both the placebo group and the angiotensin II group in ATHOS-3 (with no differences in this increase between groups), this did not translate into increased mortality for both groups; in fact, there was a trend toward improvement in survival in the angiotensin II group.

We agree with Dr. Buchtele and colleagues that the safety profile of angiotensin II should be scrutinized thoroughly, as with all novel therapies. That said, the addition of another tool in the clinician's tool box for the treatment of circulatory shock is, in our opinion, a welcome development.

Acknowledgements

Not applicable.

Funding

No funding was received.

Availability of data and materials

Not applicable.

Ethics approval and consent to participate

Not applicable.

Consent for publication

Not applicable.

\section{Publisher's Note}

Springer Nature remains neutral with regard to jurisdictional claims in published maps and institutional affiliations.

\section{Author details}

'Department of Clinical Pharmacology, Medical University of Vienna, Waehringer Guertel 18-20, A-1090 Vienna, Austria. ${ }^{2}$ Department of Emergency Medicine, Medical University of Vienna, Waehringer Guertel 18-20, A-1090 Vienna, Austria.

Received: 31 January 2018 Accepted: 21 February 2018

Published online: 16 April 2018

\section{References}

1. Busse LW, McCurdy MT, Ali O, Hall A, Chen H, Ostermann M. The effect of angiotensin II on blood pressure in patients with circulatory shock: a structured review of the literature. Crit Care. 2017;21(1):324.

2. Khanna A, English SW, Wang XS, Ham K, Tumlin J, Szerlip H, Busse LW, Altaweel L, Albertson TE, Mackey C, et al. Angiotensin II for the treatment of vasodilatory shock. N Engl J Med. 2017;377(5):419-30.

3. Antonucci E, Agosta S, Sakr Y. Angiotensin II in vasodilatory shock: lights and shadows. Crit Care. 2017;21(1):277.

4. Lamontagne F, Meade MO, Hebert PC, Asfar P, Lauzier F, Seely AJE, Day AG, Mehta S, Muscedere J, Bagshaw SM, et al. Higher versus lower blood pressure targets for vasopressor therapy in shock: a multicentre pilot randomized controlled trial. Intensive Care Med. 2016:42(4):542-50.

5. Perner A, Gordon AC, De Backer D, Dimopoulos G, Russell JA, Lipman J, Jensen JU, Myburgh J, Singer M, Bellomo R, et al. Sepsis: frontiers in diagnosis, resuscitation and antibiotic therapy. Intensive Care Med. 2016; 42(12):1958-69.

6. Busse LW, Wang XS, Chalikonda DM, et al. Clinical experience with IV angiotensin II administration: a systematic review of safety. Crit Care Med. 2017;45(8):1285-94.

7. Bassi E, Park M, Azevedo LC. Therapeutic strategies for high-dose vasopressor-dependent shock. Crit Care Res Pract. 2013;2013:654708.

8. Sviri S, Hashoul J, Stav I, et al. Does high-dose vasopressor therapy in medical intensive care patients indicate what we already suspect? J Crit Care. 2014;29(1):157-60. 